\title{
A GENERAL NOTION OF INDEPENDENCE OF SEQUENCES OF INTEGERS
}

\author{
JOHN R. BURKE
}

Gonzaga University

Department of Mathematics

Gonzaga University

Spokane, WA 99258-0001

USA

(Received February 4, 1992 and in revised form $D \div$ cember 10, 1992)

ABSTRACT. In this paper a notion of statistical independence of sequences of integers is developed. The results are generalizations of known results on independent sequences $\mathrm{mod} m$ in the integers and more generally, independent sequences on compact sets. All that is assumed is that one has a countable partition of the integers indexed by an ordered set.

KEY WORDS AND PHRASES. Independence $\bmod \mathrm{m}$, uniform distribution mod $\mathrm{m}$. 1991 AMS SUBJECT CLASSIFICATION CODE. $10 \mathrm{~K} 40$.

\section{INTRODUCTION.}

In 1940, Steinhaus and Kac [6] established the concept of independent functions on the unit square, $[0,1] \times[0,1]$. Since that time the notion of independence has been developed in several different settit.gs. The independence of sequences of integers $\bmod m$ was examined by Kuipers, Niederreiter and Shiue [2., 3]. Similar work was done by Kuipers and the author in the Gaussian integers [1]. In both of tliese situations, one of the key properties used to characterize independent sequences was the existence of a nonprincipal character on the ring structure involved. The question of what can be said about independent sequences when there is no nonprincipal character or indeed no ring structure has also been considered. Niederreiter [5] considered independence in compact spaces. In this article we examine similar questions in a more general setting. While the set $\mathbf{Z}_{\mathbf{0}}$, the nonnegative integers, is considered, any ordered countable set may be used.

\section{DEFINITIONS AND NOTATION}

Let $A=\left\{a_{n}\right\}_{n=0}^{\infty}$ be a sequence in $\mathbf{Z}_{0}$, the set of nonnegative integers. Let $\Lambda$ be a countable ordered indexing set. If $\mathrm{e}=\left\{\mathrm{C}_{\lambda}\right\}_{\lambda \in \Lambda}$ is a partition of $\mathbf{Z}_{0}\left(\mathbf{Z}_{0}=\bigcup_{\lambda \in \Lambda} \mathrm{C}_{\lambda}, C_{\lambda} \cap C_{\mu}=\varnothing\right.$ if $\left.\lambda \neq \mu\right)$, define

$$
\begin{aligned}
& \mathrm{A}\left(C_{\lambda}, n\right)=\sum_{a_{k} \in \mathrm{C}_{\lambda}} 1=\left\{\left\{a_{1}, \ldots, a_{n}\right\} \cap C_{\lambda} \mid \text {. If for each } \lambda \in \Lambda\right. \text { we have that the limit } \\
& 1 \leq \mathrm{k} \leq \mathrm{n} \\
& \lim _{n \rightarrow \infty} \frac{A\left(C_{\lambda}, n\right)}{n} \text { exists, then define } \\
& \emptyset_{\lambda}(\mathrm{A})=\lim _{n \rightarrow \infty} \frac{\mathrm{A}\left(C_{\lambda}, n\right)}{\mathrm{n}}, \lambda \in \Lambda .
\end{aligned}
$$

$\left\{\varnothing_{\lambda}(\mathrm{A})\right\}_{\lambda \in \Lambda}$ is the asymptotic distribution function (a.d.f) of $\mathbf{A}$ with respect to the partition $\mathrm{C}$. 
Now consider two sequences $A$ and $B$, both with an a.d.f. with respect to the partition $C$. Define

$$
\text { (A, B) }\left(C_{\lambda}, C_{\mu} ; n\right)=\sum_{\substack{a_{k} \in C_{\lambda}, b_{k} \in C_{\mu} \\ 1 \leq \mathrm{k} \leq \mathrm{n}}}
$$

A and B will be said to be acceptable if the limit $\lim _{n \rightarrow \infty} \frac{(\mathrm{A}, \mathrm{B})\left(C_{\lambda}, C_{\mu} ; n\right)}{n}=\varnothing_{\lambda, \mu}(\mathrm{A}, \mathrm{B})$ exists for all $\lambda$, $\mu \in \Lambda$.

Definition 1.1 Let $\mathrm{A}$ and $\mathrm{B}$ be acceptable sequences with respect to a partition $\mathrm{C}=\left\{C_{\lambda}\right\}_{\lambda \in \Lambda}$. $\mathrm{A}$ and $\mathrm{B}$ are $C$-independent provided that for all $\lambda, \mu \in \Lambda$ we have

$$
{ }_{\lambda, \mu}(\mathrm{A}, \mathrm{B})=\emptyset_{\lambda}(\mathrm{A}) \emptyset_{\mu}(\mathrm{B})
$$

If one chooses the set $\Lambda$ to be finite then $C$-independence is equivalent to that described by Niederreiter [5] in compact spaces. If the equivalence classes of the partition $C$ are chosen to be residue classes $\bmod \mathrm{m}, \mathrm{C}$-independence becomes independence $\bmod \mathrm{m}$ as considered by Kuipers, Niederreiter, and Shiue $[2,3]$.

\section{MAIN RESULTS}

THEOREM 2.1 Let $\mathrm{A}$ be a sequence which is acceptable with respect to a partition $\mathrm{e}=\left\{C_{\lambda}\right\}_{\lambda \in \Lambda}$. $A$ and $A$ are $C$-independent if and only if $\emptyset_{\lambda}(A)=1$ for some $\lambda \in \Lambda$.

PROOF: Suppose there is a $C_{\lambda}$ such that $0<\Phi_{\lambda}(A)<1$. If we assume $A$ and $A$ are $C$-independent then, since $\emptyset_{\lambda}(A)=\emptyset_{\lambda, \lambda}(A, A)$,

$$
0<\left[\emptyset_{\lambda}(A)\right]^{2}<\emptyset_{\lambda}(A)=\emptyset_{\lambda, \lambda}(A, A) .
$$

Thus A and A cannot be C-independent.

Now suppose $\emptyset_{\lambda}(\mathrm{A})=1$ for some $\lambda \in \Lambda$. Then $\emptyset_{\mu}(\mathrm{A})=0$ for $\mu \in \Lambda, \mu \neq \lambda$. It follows that for $\mu, \eta \in \Lambda, \mu \neq \lambda$ or $\eta \neq \lambda$

and

$$
\emptyset_{\mu, \eta}(\mathrm{A}, \mathrm{A})=\emptyset_{\mu}(\mathrm{A}) \emptyset_{\eta}(\mathrm{A})=0
$$

$$
{ }_{\lambda, \lambda}(\mathbf{A}, \mathbf{A})=\varnothing_{\lambda}(\mathbf{A}) \Phi_{\lambda}(\mathbf{A})=1
$$

THEOREM 2.2 Given a sequence A, A will be C-independent of B for every B such that A and B are acceptable if and only if $\emptyset_{\lambda}(A)=1$ for some $\lambda \in \Lambda$.

PROOF: From Theorem 2.1, it follows that $\emptyset_{\lambda}(A)=1$ for some $\lambda \in \Lambda$ in order that $A$ and $A$ be independent.

$$
\begin{aligned}
& \text { If } \Phi_{\lambda}(\mathrm{A})=1 \text { for some } \lambda \in \Lambda \text { and } \mathrm{B} \text { is any sequence suci: } \mathrm{A} \text { and } \mathrm{B} \text { are acceptab } \\
& \qquad \frac{\mathrm{B}\left(C_{\mu}, n\right)}{\mathrm{n}}-\sum_{\substack{\eta \in \Lambda \\
\eta \neq \lambda}} \frac{\mathrm{A}\left(C_{\eta}, \mathrm{n}\right)}{\mathrm{n}} \leq \frac{(\mathrm{A}, \mathrm{B})\left(\bar{C}_{\lambda}, C_{\mu} ; \mathrm{n}\right)}{\mathrm{n}} \leq \frac{B\left(C_{\mu}, \mathrm{n}\right)}{\mathrm{n}}
\end{aligned}
$$


Noting that

it follows that

$$
\lim _{n \rightarrow \infty} \sum_{\substack{\eta \in \Lambda \\ \eta \neq \lambda}} \frac{\mathrm{A}\left(C_{\eta}, \mathrm{n}\right)}{\mathrm{n}} \leq \sum_{\substack{\eta \in \Lambda \\ \eta \neq \lambda}} \emptyset_{\eta}(\mathrm{A})=1-\emptyset_{\lambda}(\mathrm{A})=0
$$

$$
{ }^{\emptyset_{\lambda, \mu}}(\mathrm{A}, \mathrm{B})=\emptyset_{\mu}(\mathrm{B})=\emptyset_{\lambda}(\mathrm{A}) \emptyset_{\mu}(\mathrm{B})
$$

and

$$
\emptyset_{\eta, \mu}(\mathrm{A}, \mathrm{B})=\emptyset_{\eta}(\mathrm{A}) \emptyset_{\mu}(\mathrm{B})=0 \text { if } \eta \neq \lambda
$$

As an example of the relationship between a.d.f. and the notion of $C$-independence we have the following result. We will consider the special case where $\Lambda=\mathbf{Z}_{0}$. If any other countable indexing set is used, the choice of $k$ can be done by choosing any order preserving map $f: \Lambda \rightarrow \mathbf{Z}_{0}$ and letting $k=f(\lambda)$ where $\emptyset_{\lambda}(\mathrm{A}) \neq 0$, and the subsequence $\left\{{ }^{\mathrm{a}_{k_{n}}}\right\}_{n=1}^{\infty}$ of $\mathrm{A}$ is determined by $\mathrm{a}_{k_{n}} \in C_{\lambda}$.

THEOREM 2.3 Let $A$ and $B$ be acceptable with respect to a partition $C=\left\{C_{i}\right\}_{i} \in Z_{0}$ with a.d.f. $\left\{\varnothing_{i}(\mathrm{~A})\right\}_{i \in \mathbf{Z}_{0}}$ and $\left\{\varnothing_{i}(\mathrm{~B})\right\}_{i \in \mathbf{Z}_{0}}$ respectively. Let $\mathrm{C}_{k} \in \mathrm{C}$ be such that $\emptyset_{k}(\mathrm{~A}) \neq 0$ and let $\left\{{ }^{\mathrm{a}} \boldsymbol{k}_{n}\right\}_{n=1}^{\infty}$ be the subsequence of $\mathrm{A}$ such that $\mathrm{a}_{k_{n}} \in \mathrm{C}_{k}$. If $\mathrm{A}$ and $\mathrm{B}$ are $\mathrm{C}$-independent, then the sequence $\mathrm{B}^{\prime}=\left\{\mathrm{b}_{k_{n}}\right\}_{n=1}^{\infty}$ has $\left\{\phi_{i}(\mathrm{~B})\right\}_{i} \in \mathbf{Z}_{0}$ as its a.d.f.

PROOF: For any $\mathrm{j} \in \mathbf{Z}_{0}$, since $A\left(C_{k}, k_{n}\right)=n$, we have $(A, B)\left(C_{k}, C_{j} ; k_{n}\right)=B^{\prime}(C j, n)$. By the $\mathrm{C}$-independence of $\mathrm{A}$ and $\mathrm{B}$ we have

$$
\lim _{n \rightarrow \infty} \frac{(\mathrm{A}, \mathrm{B})\left(\mathrm{C}_{k}, C_{j} ; k_{n}\right)}{k_{n}}=\varnothing_{k}(\mathrm{~A})
$$

and

$$
\frac{\mathrm{B}\left(C_{j}, n\right)}{\mathrm{n}}=\frac{(\mathrm{A}, \mathrm{B})\left(C_{k}, C_{j} ; k_{n}\right)}{k_{n}} \cdot \frac{k_{n}}{\mathrm{n}}
$$

we have, by letting $n \rightarrow \infty$

$$
\emptyset_{j}\left(\mathrm{~B}^{\prime}\right)=\emptyset_{k}(\mathrm{~A}) \emptyset_{j}(\mathrm{~B})\left[\emptyset_{k}(\mathrm{~A})\right]^{-1}=\emptyset_{j}(\mathrm{~b})
$$

Thus we have some basic properties of $C$-independent sequences as well as a method of obtaining sequences with a given a.d.f.. It would, of course, be gratifying to be able to generate acceptable

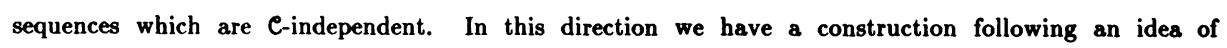
Nathanson [4].

THEOREM 2.4 Let $\mathrm{A}=\left\{a_{n}\right\}_{n=1}^{\infty}$ and $\mathrm{B}^{\prime}=\left\{b_{n}^{\prime}\right\}_{n=1}^{\infty}$ ks csceptable with respect to a partition $e=\left\{C_{\lambda}\right\}_{\lambda \in \Lambda}$ with a.d.f. $\left\{\Phi_{\lambda}(\mathrm{A})\right\}_{\lambda \in \Lambda}$ and $\left\{\Phi_{\lambda}\left(\mathrm{B}^{\prime}\right)\right\}_{\lambda \in \Lambda}$ respectively, where $\Lambda$ is $\{1,2, \ldots, \mathrm{m}\}$ or $\Lambda=\mathbf{Z}_{0}$. Then there exists a sequence $\mathrm{B}=\left\{b_{n}\right\}_{n=1}^{\infty}$ such that 
ii) $\left\{\varnothing_{\lambda}\left(B^{\prime}\right)\right\}_{\lambda \in \Lambda}$ is the a.d.f. for $B$. PROOF: Let $i_{j, k}$ denote the index of the $\mathrm{j}^{\text {th }}$ term of A contained in $C_{k}$. The sets $\left\{i_{j, k}\right\}_{j=1}^{\infty}$
partition $\mathbf{Z}_{0}$ as k runs through $\Lambda$. Define $b_{n}=b_{i_{j}, k}=\mathrm{b}_{j}^{\prime}$.

First we establish that

$$
\mathrm{B}\left(\mathrm{C}_{\lambda}, \mathrm{n}\right)=\sum_{\mu \in \Lambda} \mathrm{B}\left(C_{\lambda}, A\left(C_{\mu}, \mathrm{na}\right)\right) \text { tab } 80
$$

The relationship (1) can be seen as follows. Consider the elements $b_{k}, 1 \leq \mathbf{k} \leq \mathrm{n}$ such that $b_{k} \in C_{\lambda}$. For fixed $\mu$ we count the elements $b_{i_{1}, \mu}, b_{i_{2}, \mu}, \ldots, b_{i_{A}}\left(C_{t}, n\right), \mu$ (which are the terms $b_{1}^{\prime}, \ldots$, ${ }^{\mathrm{b}^{\prime}}{ }_{A}\left(C_{\mu}, n\right)$ ) that are in $C_{\lambda}$. There are $\mathrm{B}\left(C_{\lambda}, A\left(C_{\mu}, \mathrm{n}\right)\right)$ such terms. Now let $\mu$ run through $\Lambda$.

We now establish ii). From (*) we have

$$
\frac{B\left(C_{\lambda}, \mathrm{n}\right)}{\mathrm{n}}=\sum_{\mu \in \lambda} \frac{\mathrm{B}\left(C_{\lambda}, A\left(C_{\mu}, \mathrm{n}\right)\right)}{\mathrm{n}}=\sum_{\mu \in \Lambda} \frac{B\left(C_{\lambda}, A\left(C_{\mu}, \mathrm{n}\right)\right)}{\mathrm{A}\left(C_{\mu}, \mathrm{n}\right)} \cdot \frac{\mathrm{A}\left(C_{\mu}, \mathrm{n}\right)}{\mathrm{n}} .
$$

Letting $n \rightarrow \infty$ we have

$$
{ }_{\lambda}(\mathrm{b})=\sum_{\mu \in \Lambda} \emptyset_{\lambda}\left(\mathrm{B}^{\prime}\right) \emptyset_{\mu}(\mathrm{A})=\emptyset_{\lambda}\left(\mathrm{B}^{\prime}\right) \sum_{\mu \in \lambda} \emptyset_{\mu}(\mathrm{A})=\emptyset_{\lambda}\left(\mathrm{B}^{\prime}\right)
$$

To establish the $\mathrm{C}$-independence of $\mathrm{A}$ and $\mathrm{B}$ consider $(\mathrm{A}, \mathrm{B})\left(C_{\lambda}, C_{\mu} ; \mathrm{n}\right)=\mathrm{B}\left(C_{\mu}, A\left(C_{\lambda}, \mathrm{n}\right)\right)$ Thus

$$
\begin{aligned}
{ }_{\lambda, \mu}(\mathrm{A}, \mathrm{B}) & =\lim _{n \rightarrow \infty} \frac{(\mathrm{A}, \mathrm{B})\left(C_{\lambda}, C_{\mu} ; n\right)}{\mathrm{n}}=\lim _{n \rightarrow \infty} \frac{\mathrm{B}\left(C_{\mu}, A\left(C_{\lambda}, \mathrm{n}\right)\right)}{\mathrm{n}} \\
& =\lim _{n \rightarrow \infty} \frac{B\left(C_{\mu}, A\left(C_{\lambda}, \mathrm{n}\right)\right)}{A\left(C_{\lambda}, \mathrm{n}\right)} \cdot \frac{A\left(C_{\lambda}, \mathrm{n}\right)}{n}=\emptyset_{\mu}\left(\mathrm{B}^{\prime}\right) \Phi_{\lambda}(\mathrm{A})=\varnothing_{\mu}(\mathrm{B}) \Phi_{\lambda}(\mathrm{A}) .
\end{aligned}
$$

It would be of interest to examine how the structure of the partition $e$ affects the independence of sequences. Perhaps a partition whose classes are determined by the number of prime factors or distinct prime factors would be of interest.

\section{REFERENCES}

1. Burke, John R. and Kuipers, L., Asymptotic Distribution and Independence of Sequences of Gaussian Integers, Sim. Stev., 50 e Jaargang (1976-77), 3-21.

2. Kuipers, L. and Niederreiter, H., Asymptotic Distribution mod $\mathrm{m}$ and Independence of Sequences of Integers. I, Proc. of the Jap. Ac., Vol. 50 (1974), 256-260.

3. Kuipers, L. and Shiue, J.S., Asymptotic Distribution mod $m$ of Sequences of Integers and the Notion of Independence, Atti della Acc. Naz dei Lincei, Serie V111, Vol. XI (1970), 63-90.

4. Nathanson, M., Asymptotic Distribution and Asymptotic Independence of Sequences of Integers, Acta Math. Acad. Sci. Hungar. 29 (1977) no. 3-4, 207-218.

5. Niederreiter, H., Indépendence de suites Lecture Notes in Math., Vol. 475 Springer Berlin (1975) 54, 120-131.

6. Steinhaus, H. and Kac, M., Fonctions Indépendentes, I-VI, Fund. Math. (1936-40), 46-131. 


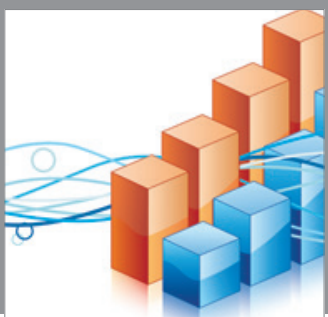

Advances in

Operations Research

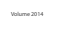

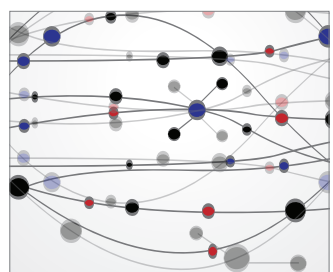

\section{The Scientific} World Journal
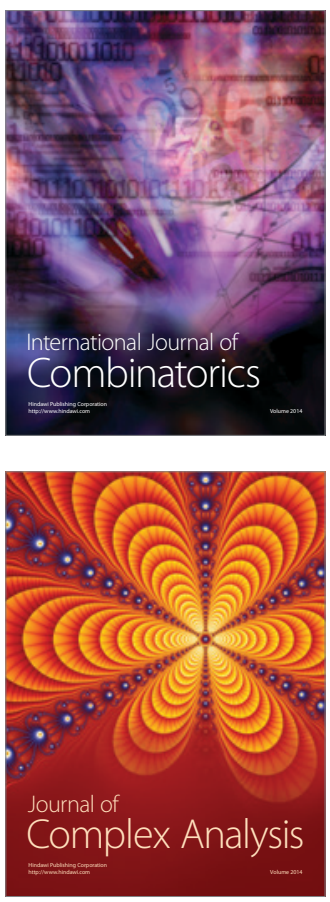

International Journal of

Mathematics and

Mathematical

Sciences
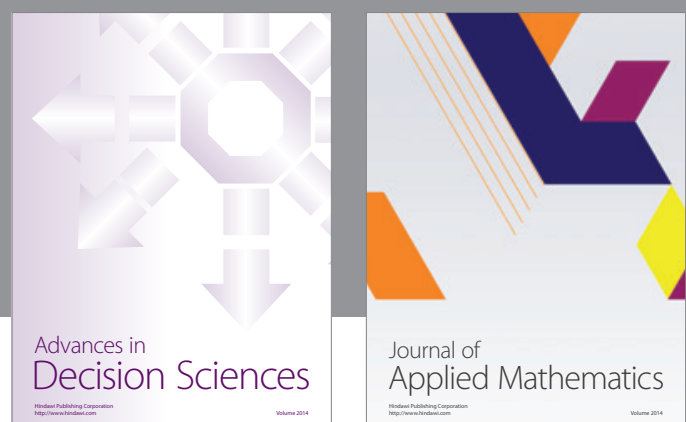

Journal of

Applied Mathematics
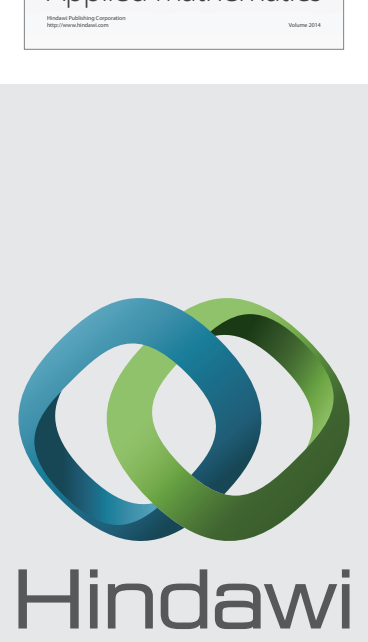

Submit your manuscripts at http://www.hindawi.com
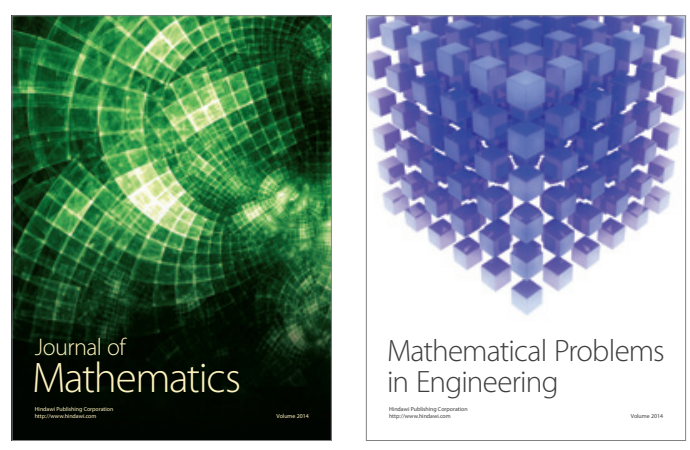

Mathematical Problems in Engineering
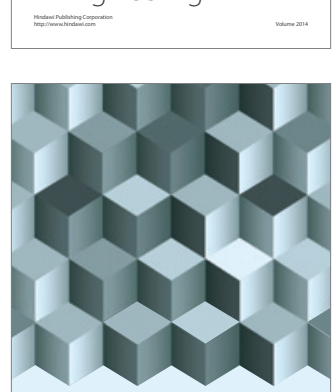

Journal of

Function Spaces
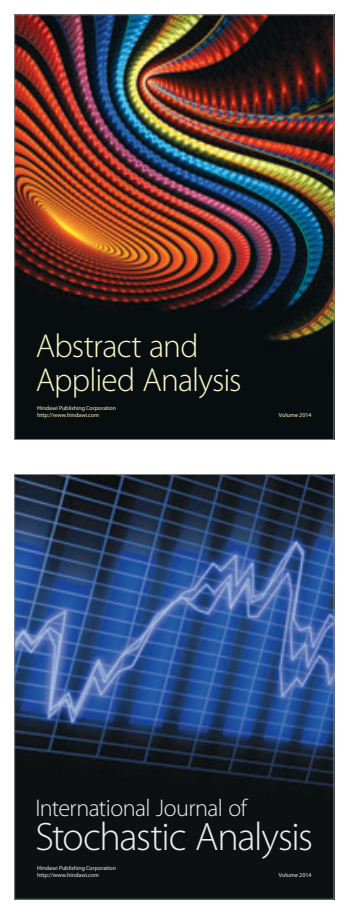

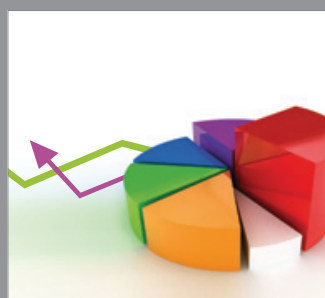

ournal of

Probability and Statistics

Promensencen
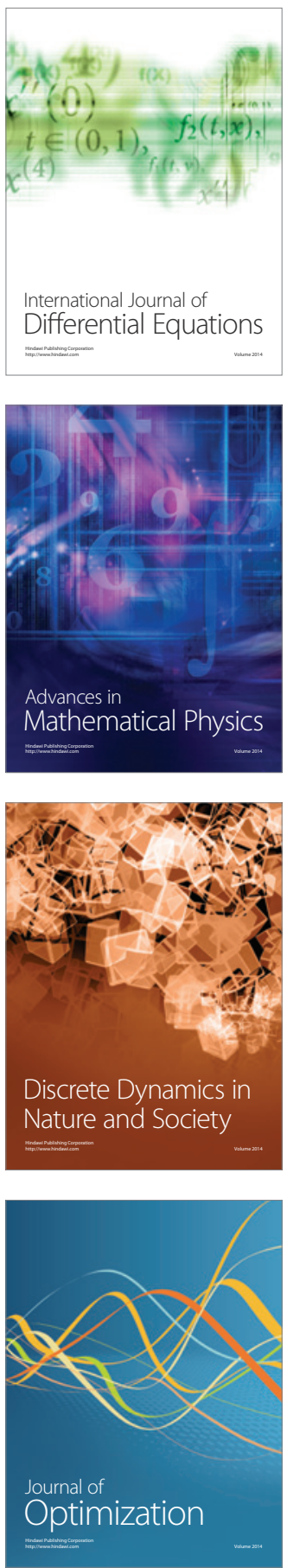